\title{
The Eye In Dystrophic Epidermolysis Bullosa: Clinical and Immunopathological Findings
}

\author{
P.J.MCDONNELL, ${ }^{1}$ \\ R.A.J.EADY ${ }^{2}$ \\ O.M.V.SCHOFIELD, ${ }^{2}$ \\ D.J.SPALTON, ${ }^{1}$ \\ B.J.MAYOU ${ }^{3}$ and
}

London

\begin{abstract}
Summary
The ocular findings in a series of patients with dystrophic epidermolysis bullosa are presented. An immunopathological technique using monclonal antibodies for examining components of the epithelial basement membrane of the conjunctiva is described and the results obtained on a conjunctival biopsy from a patient with dystrophic epidermolysis bullosa are presented.
\end{abstract}

Epidermolysis bullosa is the name of a group of inherited skin diseases which are characterised by a tendency to blister formation after minor trauma. The dystrophic form of epidermolysis bullosa results in scar formation after the blisters heal. In addition to the skin the blistering process may involve the nails, mouth, gums, teeth, oesophagus and eyes. There are a number of different types of dystrophic epidermolysis bullosa (DEB) which can be differentiated by their mode of inheritance and their clinical and pathological features. Inheritance may be autosomal dominant or recessive: the dominant cases are usually associated with mild clinical changes, whereas the recessive type often presents soon after birth and is associated with severe skin changes which lead to disabling deformity. Ocular involvement in DEB has been reported on a number of occasions but no systematic study of ocular changes has previously been described. Our initial findings were reported ${ }^{1}$ but since then we have increased the number of patients in the study and have used new immunohistochemical techniques to look at the possible mechanisms for the ocular changes.

Pathological studies have already shown that the blister formation in DEB occurs at the junction of the basement membrane of the epidermis and the underlying dermis, and electron microscopic studies have suggested that the cleavage occurs in the sublamina densa zone (Fig. 1).

The basic defect which allows the blister to develop is still unknown but recent work using monoclonal antibody probes to normal components of the basement membrane of the epidermis have identified a deficiency of some of these components in the skin of patients with DEB. ${ }^{2}$ As far as we are aware there have not yet been any studies with monoclonal antibody probes of normal conjuctiva or conjunctiva from patients with DEB.

In this present paper we report the results of a two year prospective study of the ocular findings in a series of patients with DEB, and we also report the initial results of monoclonal antibody studies on the basement mem-

From ${ }^{1}$ : Department of Ophthalmology, St Thomas' Hospital, London

and $^{2}$ : Institute of Dermatology, United Medical and Dental Schools, St Thomas' Hospital, London

and $^{3}$ : Department of Plastic Surgery, St Thomas' Hospital, London

Correspondence to: Mr P.J.McDonnell, South Wing Eye Department, St Thomas' Hospital, London SE1 $7 \mathrm{EH}$. 


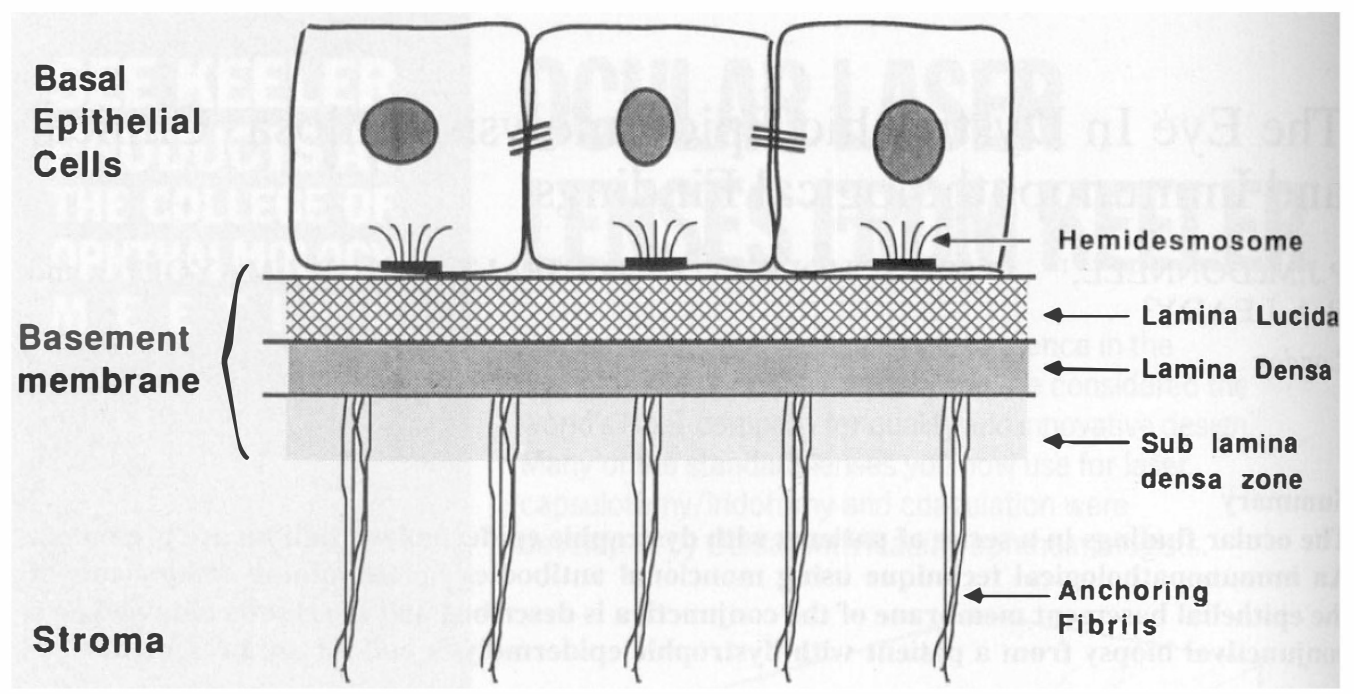

Fig. 1. Diagram showing the ultrastructure of the epithelial basement membrane.

brane of the epithelium of conjunctiva of normal patients and the conjunctiva of a patient with DEB.

\section{Patients and Methods}

Seventeen patients with DEB who were referred from the Department of Plastic Surgery or the Institute of Dermatology were reviewed in the Eye Department at St Thomas' Hospital between May 1986 and May 1988. Their mean age was 18 years with a range of 5 weeks to 55 years; there were 9 males and 8 females. All patients had a full ophthalmic assessment except for a 5 week old baby in whom slit-lamp examination was not possible. Seven of the patients were examined on more than one occasion. The results of the assessment were recorded on a special form which noted details of the type of inheritance, degree of skin involvement, and the results of the eye examination.

Small conjunctival biopsies (about $5 \mathrm{~mm}$ in diameter) were obtained from three patients with normal conjunctiva during the course of routine squint surgery and from one patient with DEB during an examination under anaesthetic. Each of these biopsies was divided in two. One half was used for electron microscopic studies and the other half was frozen in liquid nitrogen prior to labelling studies with the monoclonal antibody probe. The antibody used in this study was
LH 7:2 which is a murine monoclonal antibody raised by using an extract of trypsinised human epidermal cells as the immunogen.

The LH 7:2 antibody thus produced has been found specifically to bind with the carboxy terminal of type VII collagen which is a normal component of the lamina densa and anchoring fibriis of the epidermal basement membrane. ${ }^{3,4}$ This antibody was used to label the basement membrane of the conjunctival epithelium on cryostat sections of the biopsies by the technique of indirect immunofluorescence as described previously. ${ }^{5}$ The sections were examined using a Zeiss Standard 14 epifluorescence microscope.

\section{Results}

Fourteen of the 17 patients examined had signs of ocular involvement representing $82 \%$ of all patients examined. Seven patients $(41 \%)$ gave a history of ocular problems and the rest were asymptomatic. Six of these seven patients gave characteristic histories of recurrent corneal erosions and one had had a chalazion.

Examination confirmed the main ocular findings were as reported in our initial study. These consisted of symblepharon, limbal broadening, and corneal opacity. Symblepharon was found in 9 patients $(52 \%)$ and varied from mild changes at the medial and lateral canthi to large vascular adhesions 
between the lid margin and the cornea (Fig. 2). The limbal broadening was the commonest finding with 12 patients $(70 \%)$ having an area of broadening which either had the appearance of a vascular pannus (Fig. 3) or of a relatively avascular broadening affecting both the superior and inferior limbus. Seven patients $(41 \%)$ had corneal opacity: this was patchy and limited to the superficial stroma (Fig. 4). Four patients had a distinctive reticular pattern of opacity and three patients had a more diffuse type. All of the patients examined had taut facial skin due to scar formation following repeated blistering. In one patient this had produced some lower lid retraction and a mild degree of exposure.

The seven patients that were examined on more than one occasion demonstrated only slight changes in the ocular findings between the examinations: the longest gap between examinations was a period of one year for three patients.

Six patients in the series had had treatment for recurrent erosion in the past.

Indirect immunofluoresence microscopic examination of the LH 7:2 labelled conjunctival biopsies revealed strong linear fluoresence at the basement membrane of the conjunctival epithelium of the three control patients and no staining in the conjunctiva of the patient with DEB (Fig. 5).

\section{Discussion}

The clinical findings of this study confirm that ocular changes are very common in patients with DEB. The commonest symptom was that of recurrent erosion as has been noted in previous reports. ${ }^{6,7,8} \mathrm{~A}$ recent

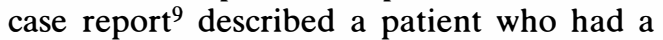
chronic corneal ulcer which needed extensive treatment including a bandage contact lens to induce healing. None of the patients in our series had chronic corneal ulcers: the corneal erosions tended to heal within 48 hours. A few of the patients who had suffered from recurrent corneal erosions for many years had devised their own managements and did not routinely seek medical attention. The three patients whom we treated for corneal erosion healed quickly.

The findings on ocular examination in this series are similar to most previous reports with symblepharon and corneal scarring being frequently observed. The exception was the appearance of limbal broadening which was actually the commonest abnormality, observed in $70 \%$ of our patients, but which has not been noted in other reports of ocular findings in patients with DEB. The

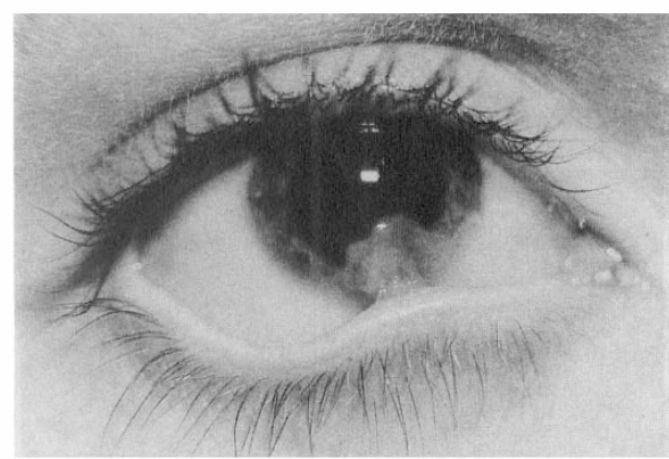

Fig. 2. Marked symblepharon with vascularisation from the lower lid to the cornea.

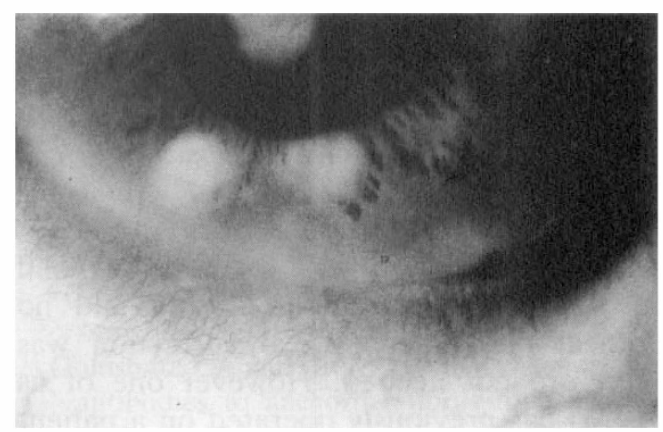

Fig. 3. Vascular broadening of the inferior limbus.

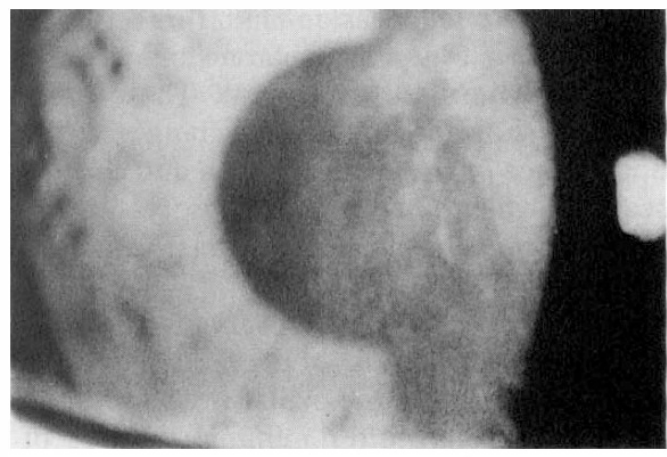

Fig. 4. Patchy corneal opacity limited to the superficial stroma. 

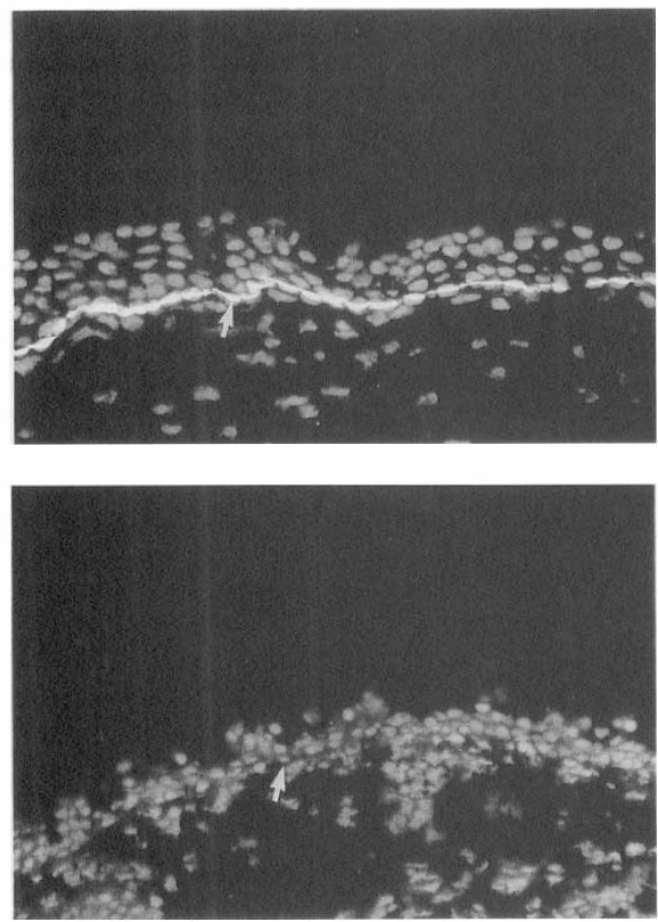

Fig. 5. Indirect immunofluoresence micrographs of conjunctiva (basement membrane ind icated with an arrow): $A$ - normal conjunctiva with intense staining with $L H 7: 2, B-D E B$ conjunctiva with no staining.

one patient who had some degree of lower lid retraction was asymptomatic and had no corneal problems: surgical correction was therefore not needed. However one of us (BJM) has previously operated on a patient with DEB to correct marked lower lid retraction using a skin graft.

Seven of our patients were reveiwed on more than one occasion and three of the patients had a repeat ocular examination a year after the first assessment. These three patients showed only slight change in the appearance of the symblepharon and corneal scarring which supports the observation by Wright ${ }^{10}$ that the changes are relatively nonprogressive.

The variety of changes observed may all be related to an instability of the epithelial basement membrane of the ocular tissues and the resulting scarring after minimal degrees of trauma. Symblepharon occurs most commonly at the canthi and thus may be related to the degree of conjunctival movement and tethering which occurs at this site. The limbal broadening may be due to long term epithelial slide related to the lid movement of blinking. Corneal scarring was often observed in the absence of an overt history of recurrent erosion and could be due to sub-clinical trauma.

Previous authors have looked at the histological changes in ocular structures of patients with DEB..$^{9,11}$ Light microscopic examination of the changes in the conjunctiva and the cornea has identified blister formation at the level of the basement membrane of the conjunctival and corneal epithelium. These findings are very similar to the changes observed in the epidermis of the skin of patients with DEB. ${ }^{12}$

Electron microscopic studies of the basement membrane of the epidermis in patients with DEB has suggested that the cleavage occurs at the level of the sublamina densa zone which is the deepest part of the basement membrane adjacent to the underlying dermis. There have not yet been reports of electron microscopic studies of conjunctiva or cornea of affected patients, however it would seem reasonable to assume that the ocular changes must also arise as a consequence of an abnormality of the conjunctival and corneal epithelial basement membrane at the level of the lamina densa. The precise mechanism of blister formation in DEB is unknown but recent work with a range of monoclonal antibody probes has identified that certain normal components of the basement membrane of the epidermis of the skin are missing from patients with DEB. ${ }^{13,14,15}$ These components lie within the lamina densa and in the underlying sublamina densa region of the basement membrane. The full significance of these abnormalities is as yet unknown: they may simply be coincidental markers for other as yet undiscovered defects at the molecular level or these abnormalities may be a vital part of the primary defect in DEB. It is possible to speculate that the absence of these normal components somehow weakens that part of the basement membrane of the epidermis and allows cleavage and blister formation to occur. As noted above, histological examination of the con- 
junctiva and cornea in affected patients shows changes similar to those in the skin. It therefore seems likely that the basic defect in the ocular tissues is also at the level of the lamina densa of the basement membrane of the conjunctival and corneal epithelium. The results of our study confirm that normal conjunctiva, like normal skin, contains a component in the lamina densa of the epithelial basement membrane which is specifically labelled by the monoclonal antibody LH 7:2. However the conjunctival sample from the patient with DEB failed to demonstrate any labelling with the LH 7:2 antibody, confirming that the basement membrane of the conjunctival epithelium was deficient of one of its components. This finding supports the hypothesis that the underlying structural defect in patients with DEB is the same in the skin and ocular tissues and consists of a deficiency of at least one component of the lamina densa of the basement membrane.

We thank Dr Irene Leigh, Consultant Dermatologist, Department of Experimental Dermatology, The London Hospital, for her kind donation of the LH 7:2 antibody.

\section{References}

${ }^{1}$ McDonnell PJ and Spalton DJ: The ocular signs and complications of epidermolysis bullosa. $J$ Roy Soc Med (in press).

2 Eady RAJ, Tidman MJ, Heagerty AHM, Kennedy AR: Approaches to the study of epidermolysis bullosa. In Honigsmann $\mathrm{H}$ ed. Current Problems in Dermatology. Basel: S Karger. 1987, 17: 127-41.

${ }^{3}$ Leigh IM, Purkis PE, Bruckner Tiderman L: LH 7:2 monoclonal antibody detects type VII collagen in the basement membrane of ectodermally derived epithelia including skin. Epithelia 1988, 1: 17-29.
${ }^{4}$ Leigh IM, Eady RAJ, Heagerty AHM, Purkis PE, Whitehead PA, Burgeson RE: Type VII collagen is a normal component of epidermal basement membrane, which shows altered expression in recessive dystrophic bullosa. $J$ Invest Dermatol 1988, 90: 639-42.

${ }^{5}$ Heagerty AHM, Kennedy AR, Leigh IM, Purkis PE, Eady RAJ: Identification of an epidermal basement membrane defect in recessive forms of dystrophic epidermolysis bullosa by LH 7:2 monoclonal antibody: use in diagnosis. Br J Dermatol 1986, 115: 125-31.

${ }^{6}$ Duke-Elder S: Systems of Ophthalmology. London: Henry Kimpton. 1965, 8: 524-7.

${ }^{7}$ Gedde-Dahl T: Epidermolysis bullosa: a clinical, genetic and epidemiological study. London: The John Hopkins Press. 1971, 64-94.

${ }^{8}$ Gans LA: Eye lesions of epidermolysis bullosa. Arch Dermatol 1988, 124: 762-4.

${ }^{9}$ Destro M, Wallow IHL, Brightbill FS: Recessive dystrophic epidermolysis bullosa. Arch Ophthalmol 1987, 105: 1248-52.

${ }^{10}$ Wright P: Cicatrising conjunctivitis. Trans Ophthalmol Soc UK 1986, 105: 1-17.

${ }^{11}$ Forgacs J and Franceschetti A: Histologic aspect of corneal changes; due to hereditary, metabolic, and cutaneous affections. Am J Ophthalmol 1959, 47: 191-202.

12 Pearson RW: Studies on the pathogenesis of epidermolysis bullosa. J Invest Dermatol 1962, 39: 551-75.

${ }^{13}$ Fine JD, Breathnach S, Hintner $\mathrm{H}$, Katz S: KF-1 monoclonal antibody defines a specific basement membrane antigen defect in dystrophic forms of epidermolysis bullosa. $J$ Invest Dermatol 1984, 82: 35-8.

${ }^{14}$ Goldsmith LA and Briggaman RA: Monoclonal antibodies to anchoring fibrils for the diagnosis of epidermolysis bullosa. J Invest Dermatol 1983, 81: 464-6.

${ }^{15}$ Heagerty AHM, Kennedy AR, Leigh IM, Eady RAJ: LH 7:2 monoclonal antibody defines a common dermo-epidermal junction defect in recessive forms of dystrophic epidermolysis bullosa (abstract). J Invest Dermatol 1985, 84: 448. 